\title{
Virtual Product Development Models: Characterization of Global Geographic Issues
}

\author{
Alyssa J. Walker ${ }^{1}$ and Jordan J. Cox ${ }^{2}$ \\ ${ }^{1}$ Brigham Young University, United States, alyssajanae@gmail.com \\ ${ }^{2}$ Brigham Young University, United States, cox@byu.edu
}

\begin{abstract}
Because product development processes now take place within a geographically diverse international community, global geographic issues relating to both physical and human elements of geography must be considered to ensure optimal deployment through means of virtual product development. Virtual product development is a strategy that preplans the product development process through simulation. Generating all possible product development process designs and simulations through virtual product development makes it possible to determine the optimal deployment option. In order to optimize in deployment, it is necessary to score each configuration based on a series of metrics that measure issues relevant to and affecting deployment. This paper presents a method by which global geographic issues may be effectively characterized in order to accurately represent their role in and influence on the product development process prior to deployment.
\end{abstract}

Keywords: globalization, graph theory, network, autonomous agents

\section{Introduction}

Due to trends of increased globalization, the product development process is no longer localized or isolated. Execution of the product development process becomes increasingly complex as a result of its deployment within a geographically diverse international community.

The spatial variation across the globe of physical features, as well as human phenomena and distribution, impacts product development in ways that are often unexpected or overlooked, but that have potential to alter greatly the final outcome of any given process in terms of cost, time, quality, etc. Geographic diversity subsequently becomes an asset or liability, depending on the deployment strategy. How then does an international corporation accurately forecast what specific geographic issues will influence its deployment, and in what ways? A method for simulating and predicting possible outcomes and forecasting the impact of geographic issues for a given product development process prior to process execution is not only beneficial but essential to successful deployment.

Please use the following format when citing this chapter:

Walker, A. J. and Cox, J. J., 2008, in IFIP International Federation for Information Processing, Volume 277; Computer-Aided Innovation (CAI); Gaetano Cascini; (Boston: Springer), pp. 119-131. 
Virtual product development is a predictive modeling technique that explores the possible product development deployment options prior to actual deployment. It is based on the use of graph models that delineate processes, organizations, and other networks relating to product development. The methodology presented in this paper is an attempt to create models for predicting the impact of global geographic issues on potential product development deployments. We recognize the limitations in attempting to represent such complex and abstract issues in a model form. However, the consequences of failing to model are apparent in the ineffective deployments of transnational corporations that exhibit themselves in current business.

\section{Literature Survey}

The characterization and modeling of globalization's impacts on businesses and organizations involve a variety of techniques and methods. Examples of these methods are presented in this section.

The modeling of complex systems such as product development deployments in a global context is a challenging undertaking. Chittaro et al define the concept of a model as "a symbolic system designed to provide a representation of a physical system appropriate for a given purpose. So, a model is only a partial representation of reality and depends on subjective decisions of the model designer" [4]. In this modeling methodology, there are two primary parts to the model: the product development deployment representation and the secondary calculus used to evaluate the deployment. The product development deployment graphs are context-independent, and the secondary calculi provide the contextdependent knowledge.

Several different groups have focused on modeling geographic issues and their impact on product development. Raper and Livingstone discuss the role of representation in modeling physical and human geography [10]. Barnes, in "Retheorizing Economic Geography" indicates the need for both a quantitative and a cultural representation [1]. Bauer states that "indeed, in going about their business, geographers often borrow from the broad suite of alternative methodologies available in other disciplines" [2]. However, in this case, we borrow from geography to construct our models of virtual product development.

The literature provides many examples of case-specific models dealing with the issues of globalization. For example, Malnight presents an evolutionary perspective by outlining the history of the transition of a multinational corporation from decentralized toward a network-based structure [8].

Tratinsky and Jarvenpaa examined the influence of global versus local contexts on information technology distribution decisions [13]. Another example of trying to model geographic issues with respect to business organizations is the work done 
by Carpenter and Frederickson. They developed a model for studying the impacts of global geographic issues of top management team characteristics [3].

The issue of developing models of geographic phenomena in the midst of globalization continues to be a controversial one. In an editorial of the Transactions of the Institute of British Geographers, the comment is made, "we need to be more self-confident and forthright in highlighting the limitations and dangers of formal modeling approaches" [6]. The editorial also states, "a meeting of minds ought to be possible: a middle ground between what it calls the "topdown' (model-building) perspective of the geographical economists and the 'bottom-up' (empirically grounded) perspective of economic geographers" [6].

Sheppard introduces the idea of scales and then indicates that the traditional hierarchy of territorial units (body, neighborhood, city, region, nation-state, supranational bloc, globe) often "splay" outward as they connect at higher scales. Additionally, Sheppard introduces networks as a way of representing the interconnections of the entities associated in globalization [11].

Subramaniam and Venkatraman recognize the need for determining product development capability [12]. Fulk and DeSanctis identify the need to represent product development in a model where issues such as communication technology can be explored [7]. Nault's approach to modeling transnational organizations incorporates the use of nodes, each of which are assigned specific quantities used in the application of a secondary calculus [9].

The work summarized in this literature survey clearly identifies the need for developing some form of a model for predicting the impact of global geographic issues on product development deployment. While it is also clear from the literature that models cannot completely represent global geographic issues, at the same time there are serious consequences for failing to model.

\section{Incorporation of Global Geographic Issues into Virtual Product Development Models}

Virtual product development (VPD) is a modeling technique used to explore before actual deployment all possible product development deployment options that are available to a company. VPD also provides a method for evaluating these options with respect to critical characteristics that have potential to impact the deployment such as time to execute, cost to execute, probability to achieve a level of quality in product offerings, etc.

Virtual product development is a modeling technique that employs graph or network models of both product development processes and the associated organizations that will execute them. Using these graphs, autonomous agents are created that represent the assignment of an actor to a given task. Agents are created for all tasks in the product development process. All possible assignments can be modeled, thus creating redundant agents. This means that there can be more than one agent who can execute a given task. All these agents are registered in a library. 
Deployment options are then determined in an autonomous agent environment where graphs of agents are reconstructed using a deterministic mapping technique. A single deployment option is a graph of agents that satisfy all tasks of the original product development process. These graphs represent unique instantiations of the original process map with different combinations of the agents. Scoring of these instantiations is accomplished through secondary calculi imposed on the graphs of the important task and actor metrics thus allowing an overall time or cost-to-execute to be computed for the given instantiation.

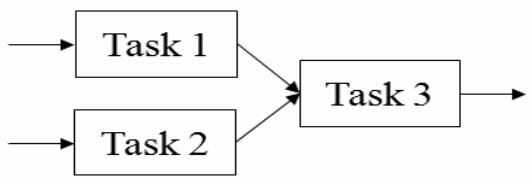

1a. A simple process graph

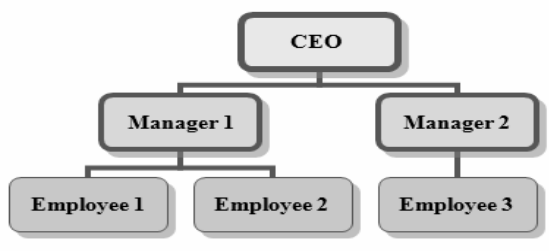

1b. A simple organization graph

Figure 1. Process and Organization graphs

In Figure 1a a simple example is shown of a process graph consisting of three tasks, two of which can be executed simultaneously (Task 1 and Task 2). Figure $1 \mathrm{~b}$ shows an example of a simple organization graph with two levels of management and three employees who will actually execute the process tasks. Assume that the overall time to execute is a critical objective for the program that will launch this process.

\begin{tabular}{|c|c|c|c|c|c|c|c|c|c|}
\hline & \multirow{3}{*}{$\begin{array}{l}\text { lime to } \\
\text { execute } \\
\text { per Skill } \\
\text { Level I }\end{array}$} & & & & & & & Agents & $\begin{array}{l}\text { Task-Actor } \\
\text { Assignments }\end{array}$ \\
\hline \multirow[t]{2}{*}{ Tasks } & & \multirow{2}{*}{$\begin{array}{l}\text { lime to } \\
\text { execute } \\
\text { per Skill } \\
\text { Level II }\end{array}$} & \multirow{2}{*}{$\begin{array}{l}\text { Time to } \\
\text { execute } \\
\text { per Skill } \\
\text { Level III }\end{array}$} & \multirow{3}{*}{ Actors } & \multirow{2}{*}{$\begin{array}{l}\text { Skill } \\
\text { level for } \\
\text { Task I }\end{array}$} & \multirow{2}{*}{$\begin{array}{c}\text { Skill } \\
\text { Level for } \\
\text { Task II }\end{array}$} & \multirow{2}{*}{$\begin{array}{c}\text { Skill } \\
\text { Level for } \\
\text { Task III }\end{array}$} & Agent 1 & Task 1-Employee 1 \\
\hline & & & & & & & & Agent 2 & Task 1 -Employee 2 \\
\hline $\begin{array}{c}1 \text { ask } \\
1\end{array}$ & 20 & 10 & 5 & & & $\pi$ & 0 & Agent 3 & Task 2-Employee2 \\
\hline $\begin{array}{c}\text { Task } \\
2 \\
\end{array}$ & 50 & 20 & 10 & $\begin{array}{l}\text { Employee } 1 \\
\text { Employee } 2\end{array}$ & II & III & 0 & Agent 4 & Task 2-Employee 3 \\
\hline $\begin{array}{c}\text { Task } \\
3\end{array}$ & 30 & 20 & 10 & Employee 3 & 0 & I & II & Agent 5 & Task 3 -Employee 3 \\
\hline
\end{tabular}

Figure 2. Enumeration of task and actor characteristics and Agent assignments

Figure 2 shows three tables, one listing each of the tasks with associated information about time to execute for three skill levels. These times to execute therefore represent the time required if an employee is qualified at a certain skill level. The second table shows each of the actors and their associated skill level for each of the tasks, and the third delineates agent assignments. These agents are created by assigning actors to tasks. Agent 1 is therefore defined as Task 1 being executed by Employee 1, Agent 2 is Task 1 executed by Employee 2 and so forth. Once all of the agent definitions are delineated, deployment options can be created. 
Figure 3 shows three deployment options made from combinations of agents which complete all of the original process tasks. Each of these deployment options can now be scored using the secondary calculus. Notice that the times are dependent upon which employee is doing what task and at what skill level. Total time scores are then based upon whether Task 1 and 2 can be done simultaneously or must be done in series because they are done by the same actor.

Deployment options

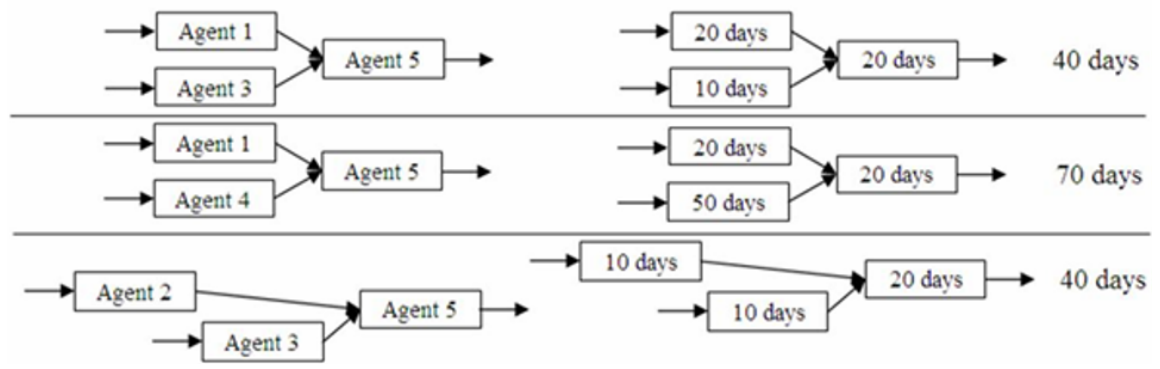

Figure 3. Deployment options and secondary calculus scores

With this in mind, notice that the time to execute for deployment option 1 is the sum of Task 1 and Task 3 since Task 2 can be executed simultaneously with Task 1. In the second deployment option, Actor 3 must execute both Task 2 and Task 3 and so the score is combined as 70 days. Finally, in deployment three, Employee 2 must complete Tasks 1 and 2 so the combined score is 40 days.

An in-depth discussion of virtual product development methods is deferred to other papers $[5,14]$. However, an overview of the techniques will be given. VPD is accomplished in several steps, each of which will be presented in the following paragraphs. The steps are as follows:

1. Identify desired product development program/project outcomes

2. Delineate process and organization models to the desired level of detail

3. Create agents by matching actors to tasks

4. Define secondary calculus to combine metrics

5. Generate possible deployment options through dynamic configuration

6. Score options

Step 1. Identify desired program/project outcomes. The first step in creating a VPD model is to select a product development process and identify desired program outcomes such as minimizing cost or time, maximizing quality, etc. There are usually one or two desired outcomes for any given program. For example, assume a company desires to launch a new product line by launching a product development process. The desired program outcomes might be profit margin, market window and quality level.

Step 2. Delineate process and organizational models to the desired level of detail. The next step is to create graph models of all the structural elements of a product development project. Structural elements include but are not limited to the 
process steps, all organizations that will contribute actors including subcontracting organizations, facilities and resource networks.

Step 3. Create agents by matching actors to tasks. The VPD simulations are created using autonomous agents. A library of all possible agents is created by assigning all qualified actors to all the possible tasks they can execute within the graph models. For example, an engineer from the parent corporation may be assigned a stress analysis task in the process. Another agent may be formed by assigning a sub-contract engineer to do the same task. All tasks must be instantiated with actors so that complete deployment options can be configured.

Step 4. Define secondary calculus to combine metrics. A secondary calculus must be defined which determines how the program objectives will be measured at each task or actor through appropriate metrics. This is done by defining trackable metrics at the actor and task level which relate to the program outcomes. For example, many of the trackable metrics will be measured in cost to the program. The secondary calculus can then be as simple as a sum across the graph of these costs. If the metrics are measures of probability that certain levels of quality can be obtained, then the calculus will be a form of Bayesian probability or Markov chains accumulated across the graph. Each set of metrics will require a different calculus based upon the type of metric and overall impact.

Step 5. Generate possible deployment options through dynamic configuration. Using autonomous agent theory and dependency mapping theory, all possible deployment options are configured. This is done by finding agents whose output are the final deliverables of the process and then satisfying their inputs with other agents in the library until all dependencies are satisfied. This produces graphs of agents representing possible deployment options. Once these graphs are produced, they can be scored using the predefined secondary calcluli.

Step 6. Score options. The secondary calculi are accumulated across the separate graphs representing deployment options. Multiple scores are then registered for the different options. These scores can then be used to select the "best" possible option. Often these deployment options are only best with respect to some criteria such as time, cost, reliability, etc.

Virtual Product Development is a modeling framework and technique that provides companies the ability to explore all possible product development deployment options before actual deployment. It also allows them to evaluate these options with respect to optimality criteria such as time, cost and quality as well as criteria based upon the impact of global geographic issues such as language differences, different government regulations, etc.

\subsection{Global Geographic Issues}

As discussed in previous sections, the shift from local to global scale inevitably carries with it a host of issues and elements with considerable potential to influence the product development process. Globalization connotes a spatial distribution, and in the context of this paper global geographic issues are characterized as any phenomenon - social, political, environmental, or otherwise - 
that influences a company's activities and varies on a global scale. The purpose of this paper is to provide a method for characterizing and incorporating these issues into VPD models, and not to identify all possible issues.

\subsection{Method of Characterization}

Once a VPD model has been developed for a given product development program, the impact of different global geographic issues on the program can be determined by expanding the existing secondary calculus. A five-step process is outlined below for converting a global issue into metrics that can be incorporated into the existing secondary calculus. For purposes of illustration, we will refer to the example used in Section 3.0 shown in Figure 1.

As shown in Figure 3, the first deployment option could be executed in forty days, the second in seventy, and the third in forty. The deployment options in reality represent different employees assigned to different sequences of tasks. For illustration of converting a global geographic issue into this secondary calculus, we choose international holidays as a global geographic issue which may affect the execution time of these deployment options. If the parent company wishes to hit a market window of March 15, 2008, they must determine when to launch the program.

Step 1. Identify the Global Geographic Issue. In this example, the global geographic issue of concern is international holidays and the impact they may have on the execution of a product development program. Obviously, this also involves the ethnic and religious backgrounds of the employees. Assume that Employee 1 is Chinese and is located in China, Employee 2 is Caucasian located in the United States, and Employee 3 is French located in France. Because the minimum time to execute the overall process is forty days, the program launch date needs to be in January, and execution will run through the February time frame. This means that at least two international holidays will occur during this program run: the Chinese New Year, which begins February 7 and will run through February 22; and Mardi Gras, which takes place February 3-5.

Step 2. Determine how Global Geographic Issue Affects Secondary Calculus Associated with the Program Objectives. Assuming that meeting the market window is a primary program objective, it is important to determine the program launch date. The actual calendar days required to execute a task must be calculated based upon time to execute the task plus the number of work days lost due to international holidays. Therefore, a new metric must be introduced into the agent secondary calculus.

Step 3. Assign Appropriate Metrics to Tasks, Actors, and Agents. Additional metrics are now required in characterizing each of the employees. Also, the agent metrics are expanded to include the number of days of impact due to international holidays for each of the tasks. In general, these metrics will be different for each type of global geographic issue and program objective. 


\begin{tabular}{|c|c|c|c|c|c|}
\hline Agents & $\begin{array}{c}\text { Task-Actor } \\
\text { Assignments }\end{array}$ & $\begin{array}{c}\text { Time for } \\
\text { Task I }\end{array}$ & $\begin{array}{c}\text { Time for } \\
\text { Task II }\end{array}$ & $\begin{array}{c}\text { Time for } \\
\text { Task III }\end{array}$ & $\begin{array}{c}\text { Holiday } \\
\text { Impact }\end{array}$ \\
\hline Agent 1 & Task1-Employee 1 & 20 & & & 15 days \\
\hline Agent 2 & Task1-Employee 2 & 10 & & & 0 days \\
\hline Agent 3 & Task2-Employee 2 & & 10 & & 0 days \\
\hline Agent 4 & Task2-Employee 3 & & 30 & & 2 days \\
\hline Agent 5 & Task3-Employee 3 & & & 20 & 2 days \\
\hline
\end{tabular}

Figure 4. Additional metrics assigned to tasks, actors, and agents.

Step 4. Collect Data that Characterizes and Measures the Appropriate Metrics. In the case of the example, data for international holidays will include identifying all of the international holidays and their duration, as well as identifying those employees who potentially would be affected by them, either because of their location or because of their affiliation. Effective characterization of global geographic issues requires the establishment of a database of metrics that have been collected, normalized, and validated. Much like the role of a company's materials database, this global geographic issues database will provide the metrics necessary for a company's modeling of product development.

Step 5. Use Data to Generate Scores based on Secondary Calculus. Using the expanded metrics, a new score for each of the deployment options is generated. As shown in Figure 5, the updated score is given in the gray circles. Notice that Deployment Options 1 and 3 were previously indistinguishable in terms of time to execute, but now are significantly different due to the impact of the international holidays. It is now obvious that Deployment Option 3 would be the optimal choice and therefore would allow program launch to occur January 17 in order to meet the March 15 market window.

Deployment options
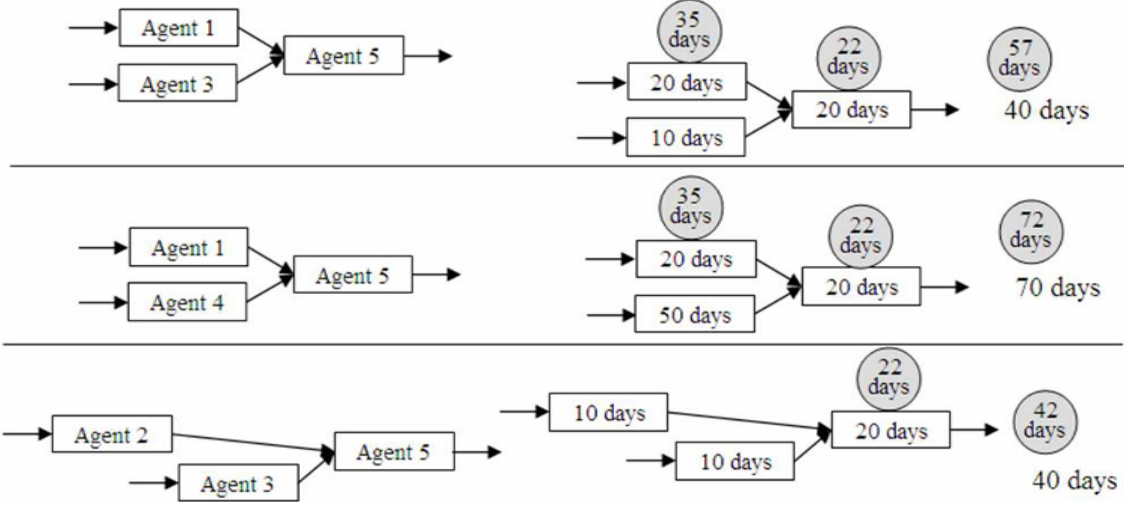

$\underline{\text { Secondary Calculus }}$
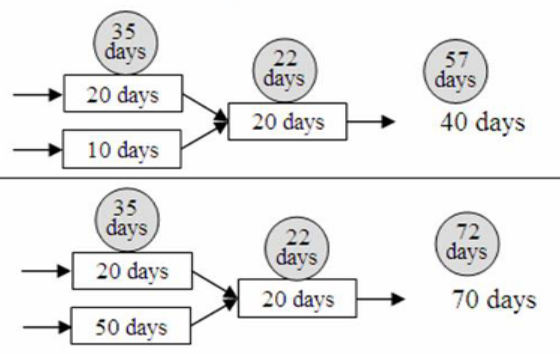

days 40 days

Figure 5. Updated process execution times based on international holidays. 


\subsection{Summary}

Complexity in virtual product development models can come from complex processes, complex combinations of actors, or complex secondary calculi. Prediction of the actual impact of a global geographic issue on a product development program that has not yet been deployed is difficult without historical data. Consequently, companies will need to develop VPD case studies that can be correlated to actual impacts to improve the validity of VPD models.

\section{Example}

A specific example from the aerospace industry involving transnational product development programs will help to illustrate not only the method, but also introduce the types of global geographic issues and extent of their impact on current product development.

Located in the western United States is an aerospace corporation specializing primarily in the engineering and manufacturing of aircraft safety devices. A recent project requiring analysis and optimization involved this aerospace corporation and a design center located in India. The project involved a total of five actors from both organizations and provides an ideal test-bed for demonstrating the methods and techniques discussed in this paper, as well as insights into specific global geographic issues affecting product development programs.

The project involved the design of a frangible housing in which thicknesses needed to be specified for optimal performance with respect to stresses in the housing. The process involved eight steps requiring the calculation and modeling of stresses, in addition to the review of the resulting models. Progress was overseen through weekly teleconferences between both sites. Specifically, the process was composed of the tasks shown in Figure 6.

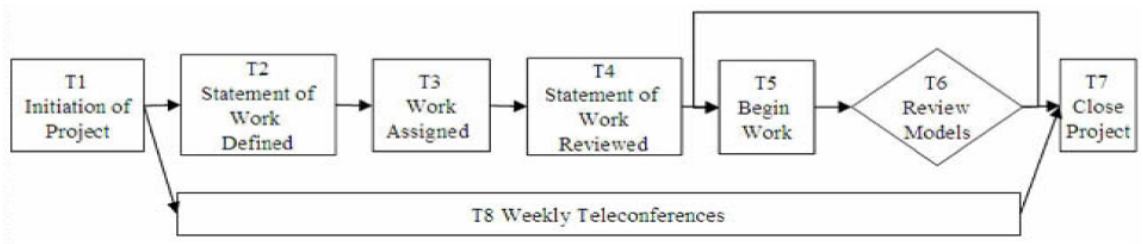

Figure 6. Sequencing of project tasks for aerospace safety project

The aerospace corporation in America made a strategic decision to acquire the design center in India in an attempt to reduce engineering costs. The decision to acquire was not necessarily based upon current need, but was motivated by the desire to establish a new company mode of operation in engineering. Consequently, direction from corporate management was given to find work that could be offloaded to the design center. This specific work project was selected because it could be completed in a relatively short amount of time and was not of critical importance to the overall program. 
In this case, only two deployment options were of interest: deployment using actors from both the design center in India and the parent company in the U.S., and deployment using solely actors from the parent company. The purpose of the VPD models was to contrast the cost and time differences between the two deployments as a means of justifying the use of the design center in India. Figure 7 therefore provides a description of each of the possible agents to be used in the two deployments.

Using the agents defined in Figure 7, there were only two deployment options available, as shown in Figure 8. Figure 8a depicts the deployment using actors from both the parent company in the U.S. and the design center in India. Figure 8b represents the deployment option using actors only from the parent company.

The scoring of each deployment option involves the calculation of two different scores which represent the sum across the graph of the metrics in the circles above each task. Both deployment options could be executed in the same amount of time and the first deployment option was $\$ 625$ less than the second. Based upon this information, the decision was made to execute using Deployment One. The project was deployed and it was discovered in the review of the work (Task 6) the wrong material properties had been used in the analysis, making the design irrelevant. The drawing had been misread due to errors in communication. Communication between engineers in the U.S. and India usually required at least a workday's cycle time due to time zone differences and limitations in direct communication.

\begin{tabular}{|c|c|c|c|c|}
\hline Agents & Task & Actors & Time to Execute & Cost \\
\hline Agent 1 & Task 1 & Actor 1 & $1 / 2$ day & $\$ 500$ \\
\hline Agent 2 & Task 2 & Actor 2 & $1 / 2$ day & $\$ 400$ \\
\hline Agent 3 & Task 3 & Actor 2 & $1 / 2$ day & $\$ 400$ \\
\hline Agent 4 & Task 4 & Actor 4 , Actor 5 & $1 / 2$ day & $\$ 200$ \\
\hline Agent 5 & Task 5 & Actor 4 , Actor 5 & 2 days & $\$ 800$ \\
\hline Agent 6 & Task 6 & Actor 2, Actor 3 & $1 / 2$ day & $\$ 800$ \\
\hline Agent 7 & Task 7 & Actor 1 , Actor 3 & $1 / 2$ day & $\$ 700$ \\
\hline Agent 8 & Task 8 & Actor 1, Actor 3 & $1 / 8$ day & $\$ 175$ \\
\hline Agent 9 & Task 4 & Actor 3 & $1 / 2$ day & $\$ 400$ \\
\hline Agent 10 & Task 5 & Actor 3 & 2 days & $\$ 1600$ \\
\hline Agent 11 & Task 6 & Actor 2, Actor 3 & $1 / 2$ day & $\$ 800$ \\
\hline Agent 12 & Task 7 & Actor 1 & $1 / 2$ day & $\$ 500$ \\
\hline
\end{tabular}

Figure 7. Agent descriptions for VPD model 


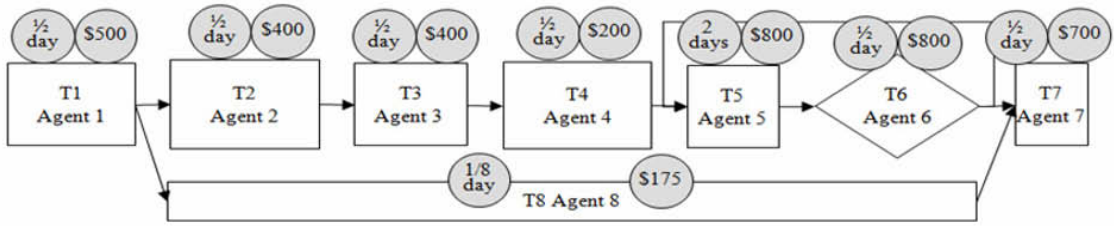

a. Deployment option $1(5+$ days, $\$ 3,975)$

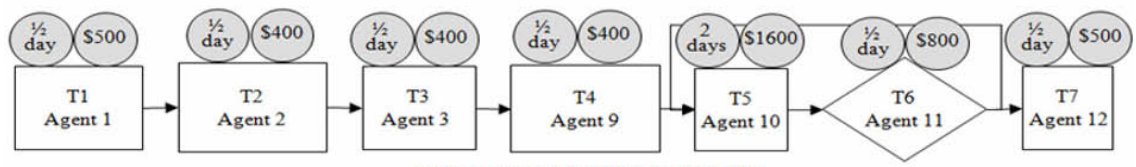

b. Deployment option $2(5+$ days, $\$ 4,600)$

Figure 8. Two VPD predicted deployment options

A second iteration of Tasks 5 and 6 was then launched to correct the errors. At the end of the second iteration, it was discovered that the right properties had been used, but the analysis had focused on the wrong portion of the product. A third iteration was launched to correct this error and finally achieved correct results in the analysis. However, part of the statement of work was to optimize thicknesses throughout the product, and the optimization focused again on the wrong portions of the product. A fourth iteration was launched to correct the errors in the optimization.

The final result of this deployment (shown in Figure 9) varied greatly from the predicted deployment option of the VPD model shown in Figure 8a. Rather than requiring $5+$ days as predicted, the process required $13+$ days to execute. The associated costs were more than two times greater at $\$ 9,300$, as opposed to the predicted $\$ 3,975$. A post-mortem analysis determined that communication limitations, time differences, lack of contextual training, incomplete instructions, and diverse training of actors contributed to the results of the actual deployment. The analysis also determined that no one group was more culpable than any other for the errors made.

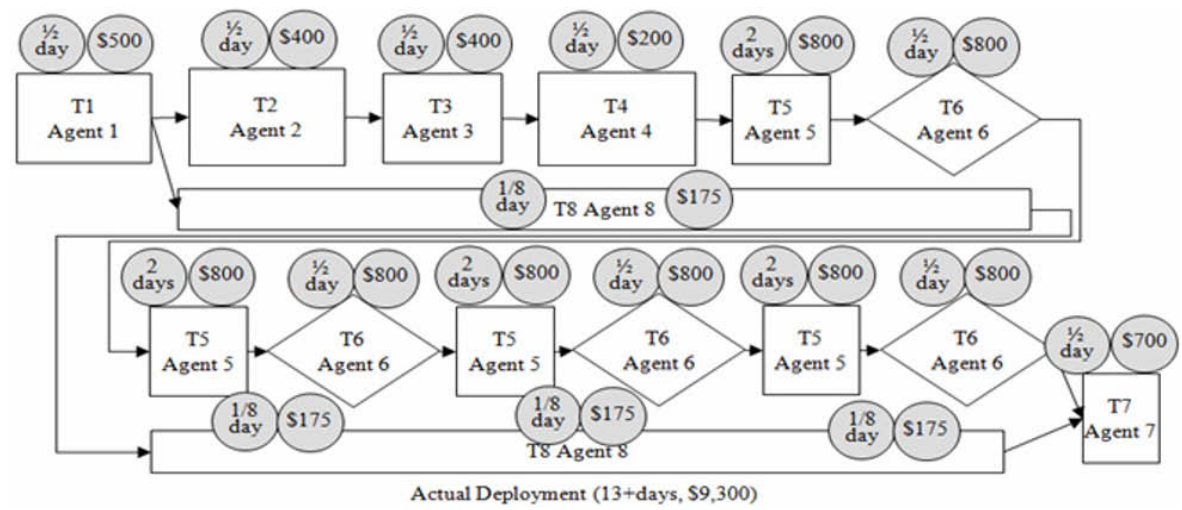

Figure 9. Actual deployment 
The nature of these errors is such that they may be classified as global geographic issues. Enumeration of each of these with a secondary calculus is difficult. Often company officials, after having executed multiple projects, calculate a factor which represents the additional time needed to execute projects such as these due to the impact of these global geographic issues. The authors have seen factors ranging from as much as 75 percent longer to 400 percent longer.

\section{Validation}

As with any modeling technique, the value of the model is dependent upon the validity of the data used. In order to create these VPD models, there is need for data relating to the global geographic issues as well as historical case studies to provide meaningful statistical correlations. Since the virtual product development models are company-specific, the identification, collection, and validation must also be company-specific and impacts must be determined within a projectspecific context.

\section{Summary and Conclusions}

Methods must be developed for leveraging global geographic issues rather than minimizing their negative impact. Virtual product development is a predictive modeling technique that explores the possible product development deployment options prior to actual deployment, generating scores for each through the use of a secondary calculus. These scores are inaccurate without the integration of global geographic issues and a quantified measure of their influence, requiring a method for the identification and characterization of such issues. That method, in addition to the need for validation and case studies, provides the focus of this paper.

\section{References}

1. Barnes, T.: Retheorizing economic geography: from the quantitative revolution to the "cultural turn," Annals of the Association of American Geographers, 91(3), 2001, p.546565.

2. Bauer, B.: On methodology in physical geography: current status, implications, and future prospects, Annals of the Association of American Geographers, 89(4), 1999, pp.677-778.

3. Carpenter, M-A.; Fredrickson, J-W.: Top management teams, global strategic posture, and the moderating role of uncertainty, The Academy of Management Journal, 44(3), 2001, 533545.

4. Chittaro, L. et al: Functional and teleological knowledge in the multimodeling approach for reasoning about physical systems: a case study in diagnosis: Transactions on Systems, Man, and Cybernetics, 23(6), 1993, pp.1718-1749.

5. Daley, J.: Autonomic product development process automation, Master's Thesis, Brigham Young University, 2007. 
6. Editorial: the 'new economic geography': challenge or irrelevance? Transactions of the Institute of British Geographers, 24, 1999, pp.387-391.

7. Fulk, J.; DeSanctis, G.: Electronic communication and changing organizational forms, Organization Science, 6(4), 1995, 337-349.

8. Malnight, T-W.: The transition from decentralized to network-based MNC structures: an evolutionary perspective, Journal of International Business Studies, 27(1), 1996, 43-65.

9. Nault, B-R.: Information technology and organization design: locating decisions and information, Management Science, 44(10), 1998, 1321-1335.

10. Raper, J.; Livingston, D.: Let's get real: spatio-temporal identity and geographic entities, Transactions of the Institute of British Geographers (24), 2001, 261-276.

11. Sheppard, E.: The spaces and times of globalization: place, scale, networks, and positionality: Economic Geography, pp.307-330.

12. Sheppard, E.: The spaces and times of globalization: place, scale, networks, and positionality: Economic Geography, pp.307-330.

13. Tractinsky, N.; Jarvenpaa, S-L.: Information systems design decisions in a global versus domestic context, MIS Quarterly, 19(4), 1995, 507-534.

14. Walker, A. J.; Cox, J. J.: Incorporating global characteristic data into virtual product development models, Journal of Computer-Aided Design and Application, 2008. 